\title{
Particle tracer response across shocks measured by PIV
}

\author{
D. Ragni $\cdot$ F. Schrijer $\cdot$ B. W. van Oudheusden • \\ F. Scarano
}

Received: 18 November 2009/Revised: 12 March 2010/Accepted: 30 April 2010/Published online: 20 May 2010

(C) The Author(s) 2010. This article is published with open access at Springerlink.com

\begin{abstract}
The experimental approach used for the evaluation of the particle response time across a stationary shock wave is assessed by means of PIV measurements. The study focuses on the experimental requirements for a reliable and unbiased measurement of the particle response time $\tau_{p}$ and length $\xi_{p}$ based on a single-exponent decaying law. A numerical simulation of the particle response experiment returns the parameters governing the measurement: namely the normalized spatial and temporal resolution, shock strength, and digital resolution. Representing the velocity decay in logarithmic coordinates it is shown that measurements performed with laser pulse separation time up to $\tau_{p}$ and interrogation window up to $\xi_{p}$ still yield unbiased results for the particle response. A set of experiments on the particle response across a planar oblique shock wave was conducted to verify the results from the numerical assessment. Liquid droplets of DEHS and solid tracer particles of silicon and titanium dioxide with different primary crystal size are compared. The resulting temporal response ranges from 2 to $3 \mu \mathrm{s}$, corresponding to values commonly reported in literature, to almost $0.3 \mu \mathrm{s}$ when particles are properly dehydrated and a filter is applied before injection into the wind tunnel. It is the first experimental evidence of particle tracers with a measured response time lower than $0.4 \mu \mathrm{s}$. The same procedure is applied to attempt the measurement of individual particle tracers by particle tracking velocimetry to estimate the spread in the distribution of tracer time response. The latter
\end{abstract}

D. Ragni $(\bowtie) \cdot$ F. Schrijer · B. W. van Oudheusden ·

F. Scarano

Faculty of Aerospace Engineering,

Delft University of Technology,

Delft, The Netherlands

e-mail: D.Ragni@tudelft.nl analysis is limited by the particle image tracking precision error, which biases the results introducing a wider broadening of the particle velocity distribution.

\section{Introduction}

The extension of PIV in high speed flows strongly relies on the ability of tracer particles to follow the flow. In the supersonic regime the presence of shock and expansion waves, where a high acceleration or deceleration occurs, causes the particle tracers to travel locally at a velocity significantly different from that of the surrounding fluid. This directly affects the accuracy of the velocity field measurement and furthermore causes the particles to be ejected towards regions with lower accelerations due to the integrated effect of particle slip as reported in many works (Lang 2000; Koike et al. 2007; Schrijer and Scarano 2007, Schrijer et al. 2008). In extreme cases the latter effect might prevent any PIV measurements due to the complete absence of particle tracers over large regions of the flow domain such as in the core of vortices and in separated flow regions. Accounts of previous studies dealing with the determination of the unsteady motion of small spherical particles in fluid flows can be found in Mei (1996), providing a general mathematical review; whereas a specific study in the high-speed regime is given by Tedeschi et al. (1999). The problem of the particle motion in turbulent compressible shear layers was treated numerically by Samimy and Lele (1991) concluding that an accurate tracing requires that the particles Stokes number should not exceed 0.1. A systematic analysis of the scattering and geometrical seeding properties and on the application of seeding PIV materials in gaseous and liquid media is provided by Melling (1997). 
In contrast, not many works have been dealing with the systematic experimental assessment of particle tracer response. Raffel et al. (2007) report from earlier studies that the particle tracer response length may easily fall below the length of the PIV interrogation size, becoming therefore not critical for PIV measurements. Lang investigated the supersonic flow around delta wings by photographic PIV and performed an accurate measurement of particle response by the shock wave test reporting a relaxation time of about $7 \mu$ s for olive oil droplets. Urban and Mungal (2001) investigated supersonic shear layers by PIV and performed oblique shock tests reporting response time of 3-4 $\mu$ s for $\mathrm{TiO}_{2}$ and in excess of $20 \mu$ s for $\mathrm{Al}_{2} \mathrm{O}_{3}$ particles. The particle velocity profile downstream of an oblique shock was spatially resolved in the PIV measurements of Scarano and van Oudheusden (2003) who inferred a relaxation time below $2 \mu$ s for $\mathrm{TiO}_{2}$ particles. The pioneering application of PIV to the hypersonic regime in shock tubes and expansion tubes (Haertig et al. 2002; and Schrijer et al. 2006) at free stream gas velocity in excess of $1,000 \mathrm{~m} / \mathrm{s}$ showed that the particle relaxation time becomes a crucial factor for the velocity measurement precision under these conditions. Later, Schrijer and Scarano (2007) proposed a method to compensate for the particle slip in hypersonic flows, based on an estimate (inferred experimentally) of the particle time response.

Inspection by electron microscopy (see for instance Raffel et al. 2007) can yield qualitative information on particles size and structure; however, the quantitative determination of the particle response time from this approach suffers from a large uncertainty due to the lack of information on the effective aerodynamic diameter and particle bulk density. Therefore, in practice, an accurate characterization of tracers response must be carried out experimentally, which eliminates the uncertainty on particle properties and fluid dynamics modeling (drag law, corrections due to rarefaction effects, etc.).

The oblique shock wave (OSW) test suits well this objective in that it provides a sharp flow velocity interface, with a uniform velocity field upstream and downstream with levels known theoretically. Previous studies have shown that this test enables the accurate evaluation of the time response $\tau_{p}$ of the particle tracers and the corresponding relaxation length $\xi_{p}$ (Melling 1997). As a consequence, the use of the OSW test has been the most commonly performed verification when attempting PIV measurements in supersonic flows. However, no systematic study is available investigating the measurement conditions that ensure a reliable and accurate estimate of the particle tracer response time.

The present work addresses in particular the choice of the most important experimental quantities affecting the reliability and accuracy of the measured particle response time, which is of primary importance in view of the developments of PIV for supersonic and hypersonic research. The study first proposes a dimensional analysis that allows identifying the parameters governing the measurement. The effect of the most important parameters to the measurement accuracy is preliminary investigated by a numerically simulated experiment. The theoretical discussion is followed by a set of experiments in a supersonic wind tunnel under well-controlled conditions, where several types of particle tracers are submitted to the OSW test. The particles vary from liquid DEHS droplets (Kähler et al. 2002) to solid $\mathrm{TiO}_{2}$ and $\mathrm{SiO}_{2}$ particles of different crystal size with and without dehydration treatment. Attention is also given to the seeding generation and two different techniques are utilized, a pressurized cyclone, and a fluidized bed system from PIVTEC GmbH. From the present experiments, particles with response time considerably below that reported in previous works could be experimentally verified.

Finally, the particle tracer size distribution and its possible biasing effect on PIV measurements are investigated. This aspect becomes relevant when seeding materials with uncontrolled properties are used in PIV experiments. The question whether the results become biased by the largest particles (more powerful scatterers) or by the more abundant small ones is frequently a matter of debate and deserves further attention. Presently, in order to understand the effect of particle tracer size on the measurement, the individual particle velocity measurement is attempted by means of a particle tracking velocimetry (PTV) approach similar to that proposed by Cowen and Monismith (1997).

\section{Background of the shock wave test}

\subsection{Particle motion across a shock wave}

The motion of a small particle immersed in a moving gas is governed by viscous drag and the inertia force (Melling 1997). The equation of motion for a spherical particle of diameter $d_{p}$, moving with velocity $\boldsymbol{V}$ while immersed in a fluid moving with velocity $\boldsymbol{U}$ and viscosity $\mu$, can be modeled as a function of the Reynolds number $R e_{p}$ based on the particle diameter $d_{p}$ :

$\frac{\mathrm{d} \boldsymbol{V}}{\mathrm{d} t}=-\frac{3}{4} C_{D} \operatorname{Re}_{p} \frac{\mu}{\rho_{p} d_{p}^{2}}(\boldsymbol{V}-\boldsymbol{U})=-K(\boldsymbol{V}-\mathbf{U})$,

where $\rho_{p}$ is the density of the particle material (assumed much heavier than the fluid density) and $C_{D}$ is the particle drag coefficient. When $K$ is assumed constant, such as for the Stokes regime, Eq. (1) admits an analytical solution for a fluid velocity undergoing an abrupt change such as across a shock wave. The result is an exponentially decaying 


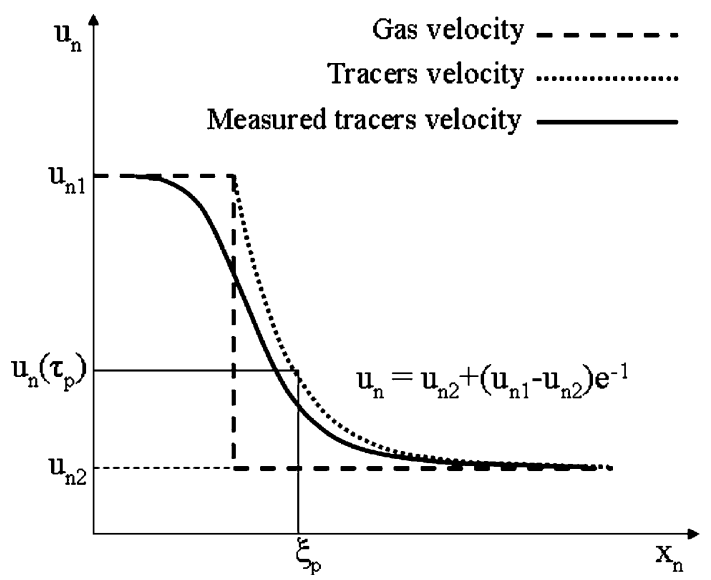

Fig. 1 Velocity profile across a shock wave. Gas velocity (dashed line), particle velocity (dotted line) and measured velocity under the effect of finite spatial and temporal resolution (solid line)

velocity of the tracer particle from the initial value towards the final reference state. This decay is characterized by a single exponent that provides the particle relaxation time $\tau_{p}$,

$\ln \left(u_{n}^{*}\right)=\ln \left(\frac{u_{n}-u_{n 2}}{u_{n 1}-u_{n 2}}\right)=-K t=-\frac{t}{\tau_{p}}$.

In Eq. (2) $u_{n}$ indicates the velocity component normal to the shock, the conditions upstream and downstream of the shock are indicated by 1 and 2, respectively. Further integration yields the particle position $x_{n}$ along the shocknormal coordinate and an explicit relationship between relaxation time $\tau_{p}$ and length $\xi_{p}$ :

$x_{n}=u_{n 2} \tau_{p} \ln \left(\frac{u_{n 1}-u_{n 2}}{u_{n}-u_{n 2}}\right)+\tau_{p}\left(u_{n 1}-u_{n}\right) \quad \Rightarrow$

$\xi_{p}=\tau_{p}\left[u_{n 1}-\left(u_{n 1}-u_{n 2}\right) \mathrm{e}^{-1}\right]$.

Most experiments are conducted in supersonic conditions at a relatively small deflection angle, rarely with a value of the normal Mach number $M_{n}$ exceeding 1.5. In these conditions, Eq. (3) is well approximated by a linear relationship between $x_{n} / \xi_{p}$ and $t / \tau_{p}$, allowing the direct computation of $\tau_{p}$ from the relaxation length measured from the spatial PIV velocity distribution.

$\ln \left(u_{n}^{*}\right)=-\frac{t}{\tau_{p}} \cong-\frac{x_{n}}{\xi_{p}}$.

\subsection{Spatial and temporal resolution effects}

The PIV technique is based on the measurement of the distance traveled by the particles within a finite time interval. Because of the finite extent of the interrogation area and the finite duration of the observation (pulse separation) the measured velocity may be considered as a spatially and temporally filtered version of the individual particle tracer velocity. The filtering effect becomes particularly visible across shocks, where the flow time scale is in the order of nanoseconds. Therefore, the spatial and temporal modulation caused by the measurement principle of PIV should be carefully considered to avoid introducing a bias error in the measurement of the relaxation time. In a realistic situation as presented in Fig. 1 the combined effect of finite spatial and temporal resolution determines the modulation of the velocity profile at the shock location.

To investigate the effect of spatial and temporal modulation, two non-dimensional ratios are introduced, the spatial ratio SR and the temporal ratio TR normalizing the interrogation window size WS and the pulse separation time $\Delta t_{p}$ with the particle relaxation length and time, respectively:

$\mathrm{SR}=\frac{\mathrm{WS}}{\xi_{p}} ; \quad \mathrm{TR}=\frac{\Delta t_{p}}{\tau_{p}}$.

Particles traveling across a shock wave are simulated by a computer and synthetic images are generated. The analysis is performed varying SR and TR between 0.1 and 4. An increase of the temporal ratio beyond the unit value introduces a significant distortion in the exponential decay (Fig. 2, left). Moreover, an apparent upstream shift of the shock location is introduced. The latter is due to the interrogation scheme by cross-correlation based on window shift (or deformation) by a central scheme (Wereley and
Fig. 2 Effect of temporal ratio on the measured velocity profile $(\mathrm{SR}=0.5): u_{n}^{*}$ in linear $($ left $)$ and logarithmic scale (right)
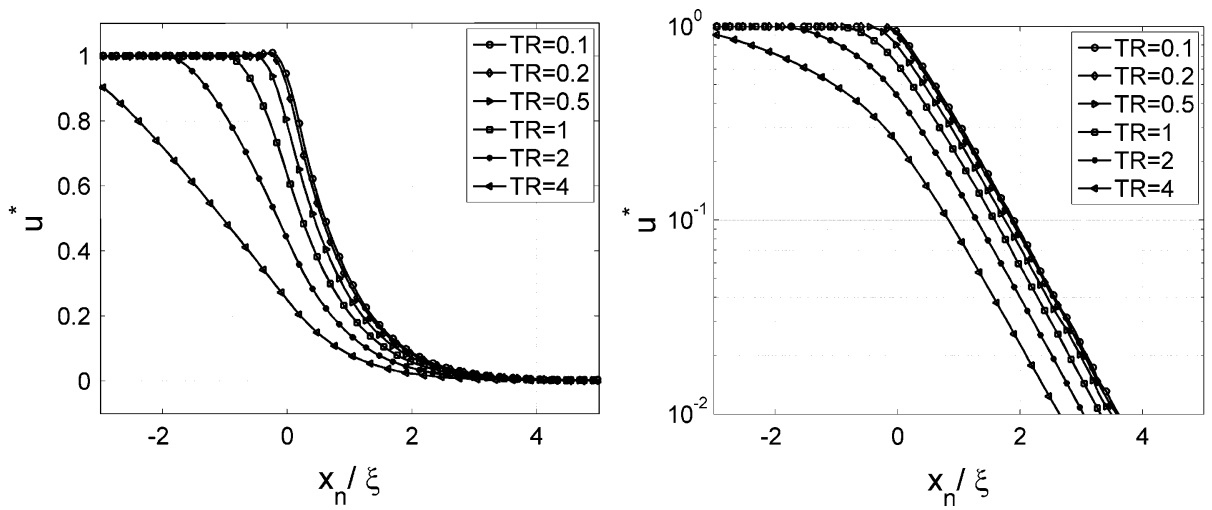
Meinhart 2001). In fact due to the finite time interval, particles falling downstream of the shock at the second exposure will result in a decelerated flow even if the vector (located at the centre of the window) falls upstream of the shock. The representation in logarithmic scale (Schrijer and Scarano 2007) is given in Fig. 2, right and offers a clear advantage for the accurate determination of the relaxation time even for measurements performed at a TR $>1$ (long pulse separation time with respect to the particle relaxation time). As a result, the correct slope is exhibited downstream, provided the data are evaluated at sufficient distance from the shock location, which allows an unbiased evaluation of $\xi_{p}$ and in turn $\tau_{p}$. For instance, a measurement performed at $\mathrm{TR}=0.5$ returns useful data for $u_{n}^{*}<0.9$. In contrast, for $\mathrm{TR}=2$ the valid data range begins at $u_{n}^{*}<0.25$, which reduces the dynamic range of the measurement.

The situation is slightly different for the spatial ratio SR. The finite extent of the interrogation window introduces an increasing symmetrical filtering effect (spatial modulation) when SR increases (Fig. 3). The effect becomes important when SR $>2$. Remarkably, temporal and spatial ratios of about 1 still offer a considerable part of the logarithmic plot to perform a reliable shock wave test. The performed numerical analysis shows that in a PIV experiment, the conditions $\mathrm{SR}, \mathrm{TR} \leq 1$ may be considered acceptable for an accurate measurement of the tracer response time. This condition has implications on the required seeding concentration and digital imaging resolution as well as on the time separation between exposures. Currently, CCD cameras for PIV are equipped with full-frame interline transfer architecture and interframe below the microsecond. However, for the study of particle tracers with a relaxation time of a fraction of $5 \mu \mathrm{s}$, the measurements may be carried out in doubleexposure mode. Concerning the limitations in terms of spatial resolution, it may be recalled that a high seeding density needed to perform measurements at submillimeter scale could be avoided if the velocity field is performed by time-averaging the correlation signal (ensemble-correlation, Meinhart et al. 2000).

\subsection{Measurement dynamic range effects}

Together with the temporal and spatial ratio, the accurate measurement of the time response requires that the velocity difference across the shock is significantly larger than the measurement error committed with the PIV measurement. The precision error is commonly referred to be a fraction of a pixel (typically 0.1 pixels for instantaneous measurements, Westerweel 1997). However, the measurement accuracy is not limited by the precision error when addressing ensemble averages or when the ensemble-correlation average technique is adopted. In these cases the limit in the accuracy is set by the bias error, which may be smaller than 0.01 pixels, especially when advanced interrogation algorithms are adopted. The velocity dynamic range further depends upon the digital imaging resolution DR (pixels $/ \mathrm{mm}$ ), which determines the number of pixels traveled by a particle image for a given time separation. Furthermore, for a given choice of these three measurement parameters the velocity ratio across the shock is a function of the normal Mach number $M_{n}$.

A synthetic view of the effect of the four mentioned parameters to the measurable range within the relaxation region is given in Fig. 4, left. The available data range is limited from the left (shock location) by the finite temporal and spatial resolution and from the bottom by the measurement accuracy and to a lesser extent by the uncertainty on the flow velocity downstream of the shock. Increasing the temporal ratio moves the range to the right in the shock region and towards the bottom in the relaxation region. Therefore, the measurement may be rather indifferent to changes in TR. Instead, an increase of digital resolution DR widens the valid data range from the bottom. The same effect is obtained increasing the normal Mach number. However, this introduces also more pronounced aerooptical aberrations and large inhomogeneity in seeding
Fig. 3 Effect of spatial ratio on the measured velocity profile $(\mathrm{TR}=0.5): u_{n}^{*}$ in linear and logarithmic scale
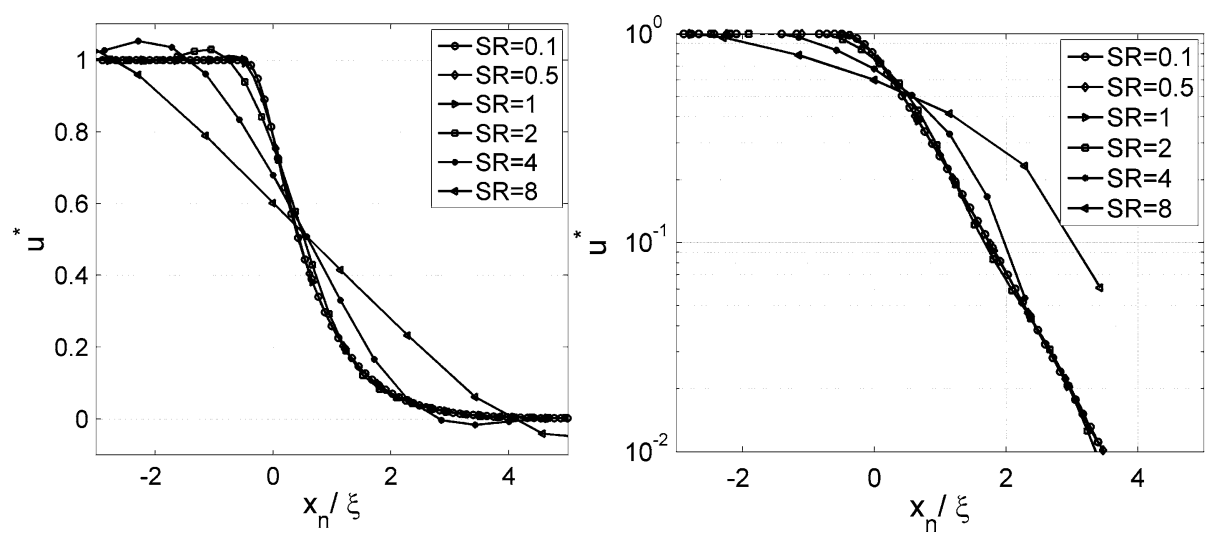
Fig. 4 a Valid data range according to measurement parameters; $\mathbf{b}$ dependence of the minimum measurable velocity on the shock strength $\left(M_{n}\right)$ for different values of the displacement upstream of the shock

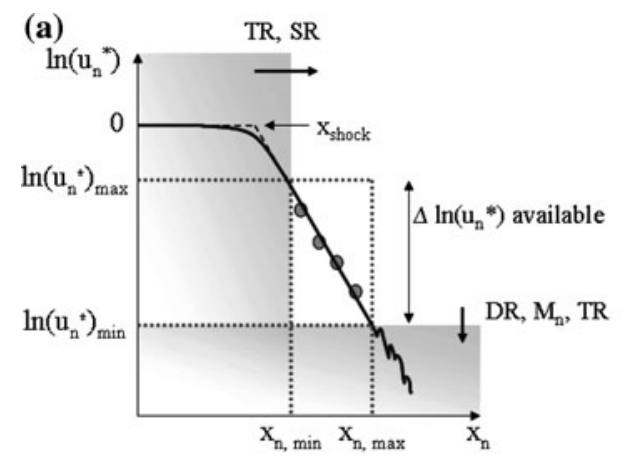

Table 1 Shock wave experiment parameters and resulting measurement accuracy

\begin{tabular}{lll}
\hline & $\tau_{p}=4 \mu \mathrm{s}$ & $\tau_{p}=2 \mu \mathrm{s}$ \\
\hline PIV measurement parameters & & \\
$F O V[\mathrm{px}, \mathrm{mm}]$ & $1,376 \times 1,040,7.9 \times 6.0$ \\
$D R[\mathrm{px} / \mathrm{mm}]$ & 170 & \\
$M, M_{n}$ & $2,1.25$ & \\
$\mathrm{SR}\left(\tau_{p}=4 \mu \mathrm{s}, \tau_{p}=2 \mu \mathrm{s}\right)$ & 0.1 & 0.2 \\
TR $\left(\tau_{p}=4 \mu \mathrm{s}, \tau_{p}=2 \mu \mathrm{s}\right)$ & 0.2 & 0.3 \\
Parameters related to the accuracy & & \\
$u_{n \infty}, \Delta u_{n}[\mathrm{px}]$ & 15,5 & 31,9 \\
$\varepsilon\left(u_{n \infty}\right)[\mathrm{px}, \%]$ & $0.1,0.3 \%$ & $0.05,0.2 \%$ \\
$\ln \left(u_{n l i m}^{*}\right)$ & -1.9 & -1.9 \\
$\Delta \ln$ required & 1.5 & 1.6 \\
$N$ uncorr. & 4 & 12 \\
$\varepsilon_{\tau}=\sigma(p=2)$ & $7 \%$ & $3 \%$ \\
\hline
\end{tabular}

density, which effects are not included in the present discussion.

The relation between $M_{n}$ and the minimum measurable velocity $u_{n}^{*}$ (for a given choice of the particles displacement in pixels, viz. for different values of DR) is illustrated in Fig. 4, right. The most significant improvement is obtained in the range of $M_{n}$ between 1 and 1.5. A further increase does not yield significant reduction of the minimum measurable velocity difference.

The required $\Delta \ln \left(u_{n}^{*}\right)$ has to be inferred from velocity measurements at two different spatial locations. When more measurement points are available, a least-squares fit may be used, with an accuracy depending upon the precision of each data-point as well as their number $N$, In turn, depending on the spatial ratio SR. Following the approach of Draper and Smith (1982) in the analysis of a linear least squares regression on an ensemble of $N$ data, the variance $\sigma^{2}$ on the fit depends on the single error terms in the regression. To verify the validity of a determined set of parameters the velocity measurement range $\Delta \ln \left(u_{n}^{*}\right)$ must be compared to the expected measurement uncertainty, quantified by the variance $\sigma$. For example, a measurement of $\tau_{p}$ with uncertainty

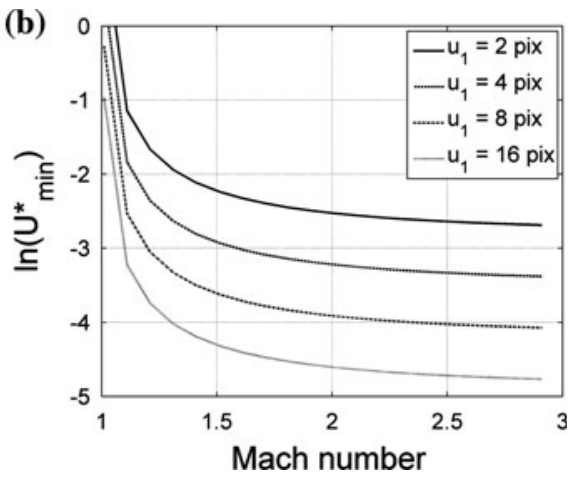

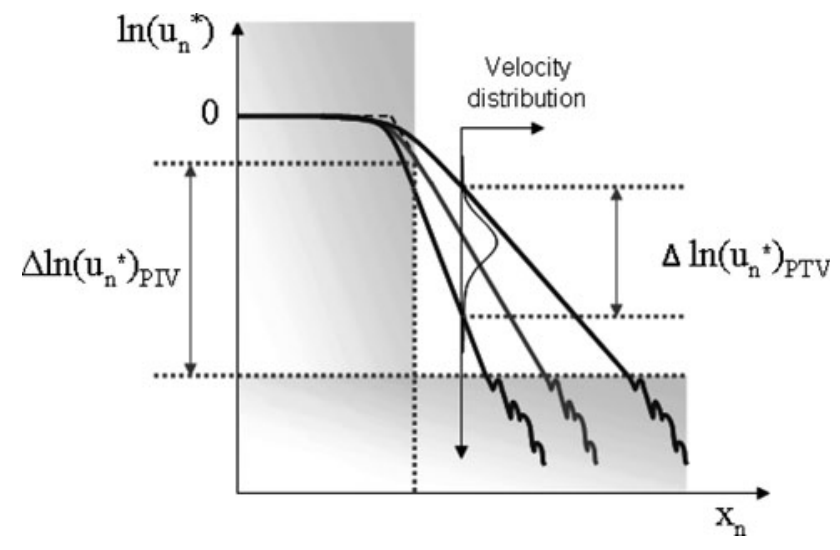

Fig. 5 Expected broadening for particle tracers of different response and available data range for the PTV analysis

within $5 \%$ requires 8 points and a velocity measurement with error smaller than 0.1 pixels $(0.3 \%)$. In Table 1 relevant values are summarized, where some parameters are based on the experiments performed in the present study.

The foregoing analysis may be extended to the case of individual particle velocity measurement. However, an accurate estimate of the time response is constrained by the higher measurement errors associated with the precision error of the instantaneous velocity measurement from individual particle tracking. This error is typically in the order of 0.3 pixels (Cowen and Monismith 1997) and cannot be reduced by data averaging. This causes a significant reduction of the valid data range due to the increased minimum measurable velocity (from the bottom). Moreover, when particles with different response time are to be identified, the target of the measurement becomes the difference between the different relaxation paths, which further reduces the dynamic range (vertical difference indicated in Fig. 5).

\subsection{Disturbing factors: aero-optical aberrations and unsteady flow effects}

The measurement of the tracer motion across the shock can be compromised by aero-optical aberrations (Elsinga et al. 
2005). The presence of the shock wave is a refractive interface and under specific conditions particle image result blurred causing a sudden drop of correlation signal in the shock region. When the viewing direction intersects the shock from a small angle upstream (typically $2^{\circ}-5^{\circ}$ as suggested by Elsinga et al. 2005) this phenomenon is largely reduced. The predominant $2 \mathrm{D}$ nature of the flow avoids in this case the introduction of any bias error due to the inclined view. Additionally, the extent of blur is reduced by increasing the numerical aperture $f \#$ of the imaging system. Despite these measures, for the given shock strength and optical magnification, aero-optical distortion was still detectable along a region where the particle images appear slightly deformed (Fig. 6). Note that the rotation of the FOV with respect to the conventional horizontal-vertical reference system is not related to the aforementioned tilting. The rotation of the FOV enables to image the oblique shock aligned with the image frame of reference, which improves the spatial resolution when elongated rectangular windows are used for the analysis.

The requirements imposed on spatial resolution imply that any shock movement (due to small variations in the free-stream Mach number) or displacement of the field of view (due to vibrations of the imaging system) has to be minimized or compensated afterwards by realigning the images. For this purpose the shock location is estimated making use of the blurred line. The latter is identified by computing the auto-correlation and detecting where the peak broadens, as already proposed by Elsinga et al. (2005). In the current experiments a shock motion in a range of approximately 100 pixels has been reduced to $<20$ pixels after realignment. An additional measure to avoid the shock motion due to free-stream Mach variations is to measure very close to the leading edge of the shock generator.

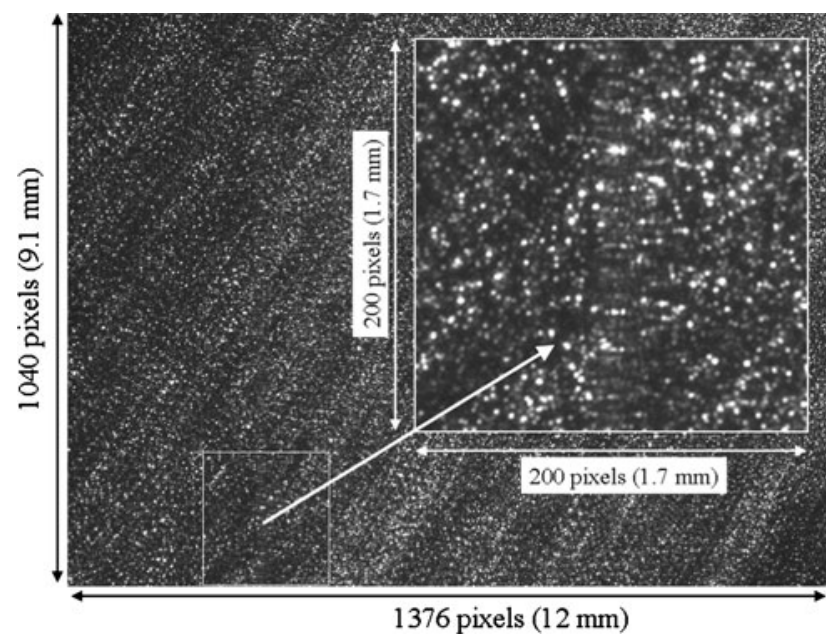

Fig. 6 Double-exposure PIV recording showing evidence of some aero-optical aberration

\section{Experimental apparatus and procedure}

\subsection{Wind-tunnel and model}

Experiments were performed in the ST-15 supersonic blow-down wind-tunnel in the Aerodynamics Laboratories of the Aerospace Engineering Department of TU Delft. The wind tunnel test section is $16 \times 15 \mathrm{~cm}^{2}$ and the tunnel was operated at Mach 2. The conditions in the settling chamber are $P_{0}=3.4 \mathrm{bar}$ and $T_{0}=275 \mathrm{~K}$. A two-dimensional wedge of $13.0 \mathrm{~cm}$ span imposes a flow deflection $\theta=9^{\circ}$ and an OSW inclined at $37.2^{\circ}$ is formed.

\subsection{PIV apparatus and data analysis}

The particle tracers are illuminated by a Big Sky CFR-PIV$200 \mathrm{Nd}$ :Yag laser $(200 \mathrm{~mJ}$ pulse energy, $7 \mathrm{~ns}$ pulse duration, $532 \mathrm{~nm}$ wavelength). A light sheet of approximately $1.5 \mathrm{~mm}$ thickness is formed at the measurement location. The light scattered by the particle images is recorded with a LaVision Imager Intense CCD camera $(1,376 \times 1,040$ pixels, 12 bits, $6.45 \mu \mathrm{m}$ pixel pitch). The highest resolution was obtained using a Carl Zeiss Jena objective $(f=120 \mathrm{~mm})$ detached from the camera in order to reach a magnification factor of $M=1.12$ yielding a FOV of $7.9 \times 6.0 \mathrm{~mm}^{2}$ and a digital resolution of 174 pixels $/ \mathrm{mm}$. The system is operated in either single-exposure-doubleframe or double-exposure mode, with separation from $800 \mathrm{~ns}$ down to $400 \mathrm{~ns}$. The laser control, camera synchronization, and image acquisition are performed by a PC equipped with a LaVision programmable timing unit (PTU8) controlled by DaVis7.2.

The vertical axis of the FOV is aligned with the shock, which greatly simplifies the data averaging along the shock and partly contributes to a higher measurement spatial resolution (the interrogation windows can be aligned and stretched along the shock direction). The line of sight is tilted of $4^{\circ}$ with respect to the measurement plane normal to reduce optical aberration effects. Series of 200 images are recorded at a rate of $10 \mathrm{~Hz}$. The recorded images are evaluated with the ensemble auto-correlation technique (Meinhart et al. 2000) available in DaVis7.4, yielding the ensemble-averaged velocity of the imaged particle tracers. The PTV analysis was performed by a custom-made software based on the super-resolution concept (Keane et al. 1995). Particle detection and pairing are based on the recipes provided by Cowen and Monismith (1997).

\subsection{Flow seeding}

Experiments are conducted with liquid particles and a variety of solid tracers with different primary crystal size. The specifications of the materials used are summarized in 
Table 2 Seeding materials and particles physical properties

\begin{tabular}{lllc}
\hline $\begin{array}{l}\text { Seeding } \\
\text { identification }\end{array}$ & Material & $\begin{array}{l}\text { Primary crystal } \\
\text { size }(\mathrm{nm})\end{array}$ & $\begin{array}{l}\text { Bulk density } \\
(\mathrm{g} / \mathrm{l})^{\mathrm{a}}\end{array}$ \\
\hline Degussa Aerosil R104 & $\mathrm{SiO}_{2}$ & 12 & $\sim 50$ \\
Degussa Aerosil R972 & $\mathrm{SiO}_{2}$ & 16 & $\sim 50$ \\
Kemira P170 & $\mathrm{TiO}_{2}$ & 14 & 150 \\
Kemira P580 & $\mathrm{TiO}_{2}$ & 30 & 150 \\
Kemira L830 & $\mathrm{TiO}_{2}$ & 50 & 200 \\
Kemira 300 & $\mathrm{TiO}_{2}$ & 170 & 800 \\
XRDI-S & $\mathrm{TiO}_{2}$ & 240 & 800 \\
DuPont R931 & $\mathrm{TiO}_{2}$ & 550 & 3,636 \\
\hline
\end{tabular}

a Tapped density for Aerosil particles

Table 2. Solid tracer particles have been tested before and after dehydration by exposure to about $130^{\circ} \mathrm{C}$ for $2 \mathrm{~h}$ in a non-ventilated temperature-controlled system (Fig. 7).

Two solid seeding generators have been used: a custommade cyclone seeder (Fig. 9, left) and a fluidized bed from PIVTEC GmbH (Fig. 9, center). The first has a diameter of $7 \mathrm{~cm}$, height of $12.5 \mathrm{~cm}$ with a pipe of $4 \mathrm{~mm}$ diameter located at $6 \mathrm{~cm}$ from the head and $3 \mathrm{~cm}$ from the axis. The air enters the chamber through a tangential inlet producing a flow that entrains the particles on the surface. The seeded air is injected upstream of the settling chamber, providing a homogeneous dispersion in the test section (see Fig. 8). The system was operated at 4.5 bar ( 1 bar overpressure with respect to the settling chamber pressure) with the cyclone and at 7.5 bar with the PIVTEC system; the first equipped with or without an additional particle filter (3 M 2135, AS/NZS 1716 P3).

Liquid droplets of di(2-ethylhexyl) sebacate (DEHS) seeding have been tested substituting the cyclone and the rake with a the PIVTEC PIVpart 45 seeding generator equipped with 12 Laskin nozzle delivering droplets of $1 \mu \mathrm{m}$ median diameter (Kähler et al. 2002).

\section{Results and discussion}

\subsection{Mean relaxation time from PIV analysis}

Time-averaged velocity fields across the oblique shock are obtained from 200 instantaneous measurements. The measurements performed with DEHS particles are illustrated in Fig. 10 by contours of shock-normal velocity component.

The normal velocity profile across the shock is obtained by further data averaging along the shock direction (after data realignment when necessary) to improve the statistical convergence. Each velocity measurement in the shock normal diagram is obtained from approximately 20,000 samples. The resulting diagrams are presented in Fig. 11, where the data points span from upstream the shock to approximately $4 \xi_{p}$ downstream of it. The relaxation length $\xi_{p}$ is obtained from the data points in the range of $\ln \left(u_{n}^{*}\right)=[-1.5 ;-0.5]$ by means of a least squares linear fit.

The relaxation time for the investigated seeding materials is summarized in Table 3. The DEHS droplets show a time response of $2 \mu \mathrm{s}$; however, a significant data scatter (400 ns) is observed, which is ascribed to the increased measurement uncertainty due to a relatively weak light scattering. This was partly compensated decreasing the $f \#$ from 11 to 8 . The results of two different experiments are presented in Fig. 11, indicating the typical level of repeatability. For the solid particles, the relaxation time ranges from 0.4 to $3.7 \mu$ s. The data scatter is in the order of 100 ns. Further investigation on the solid materials suggested that the effect of a prolonged storage cannot be fully eliminated by the drying procedure and large agglomerates are still visible in the PIV images. In all the analyzed conditions the velocity diagram is rounded at the shock location due to spatial and temporal averaging, to an extent

Fig. 7 Test section, illumination and imaged region

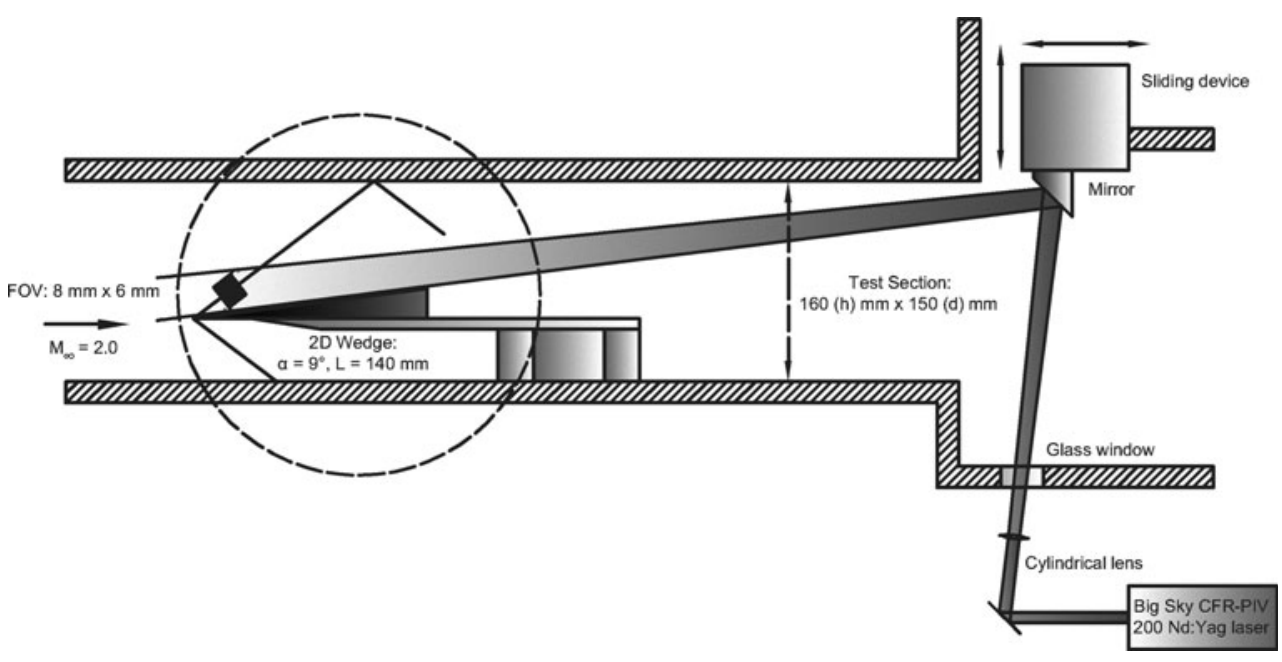


Fig. 8 Wind tunnel and seeding system schematics

Fig. 9 Seeding generation devices. TU Delft cyclone (left); PIVTEC fluidized bed (center), PIVTEC DEHS atomizer (right)
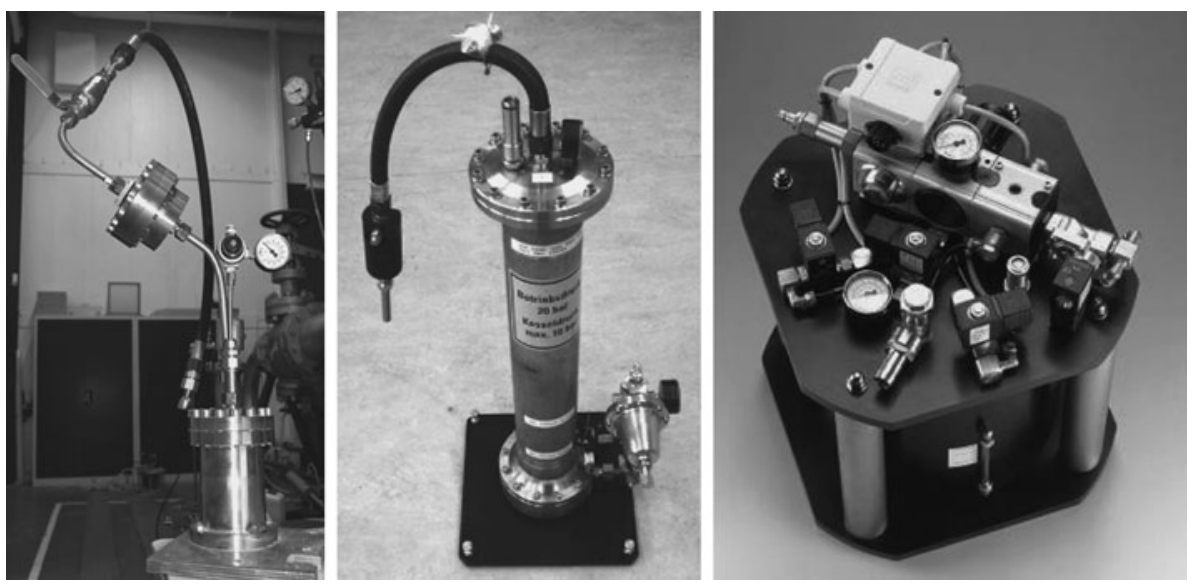

Wind-tunnel test section: Mach 2 nozzle

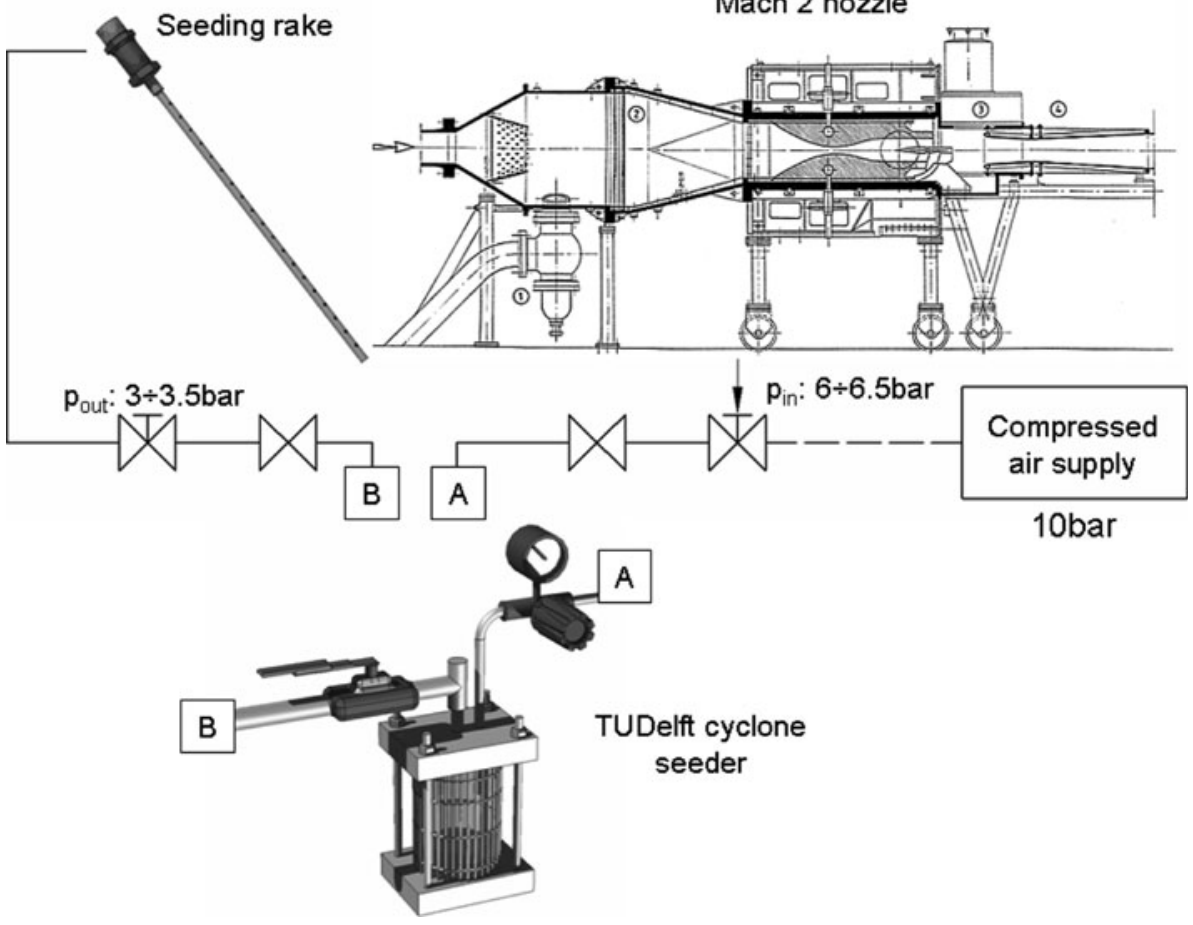

Fig. 10 Measured normal velocity component across the shock in a single snapshot (left) and in the average field (right) for $\mathrm{TiO}_{2} 50 \mathrm{~nm}$
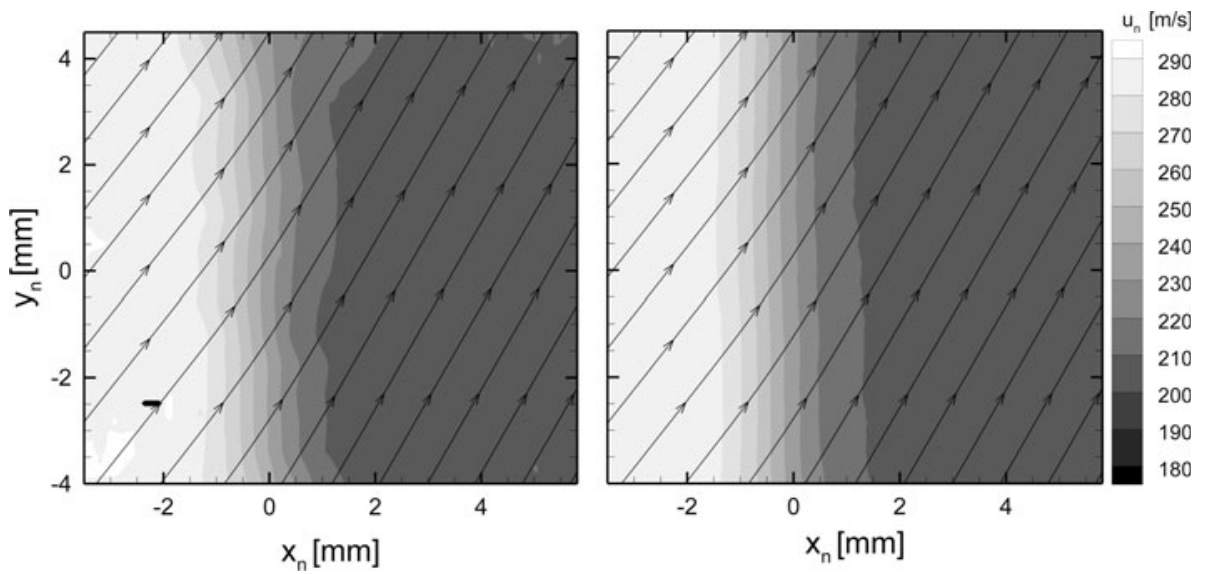

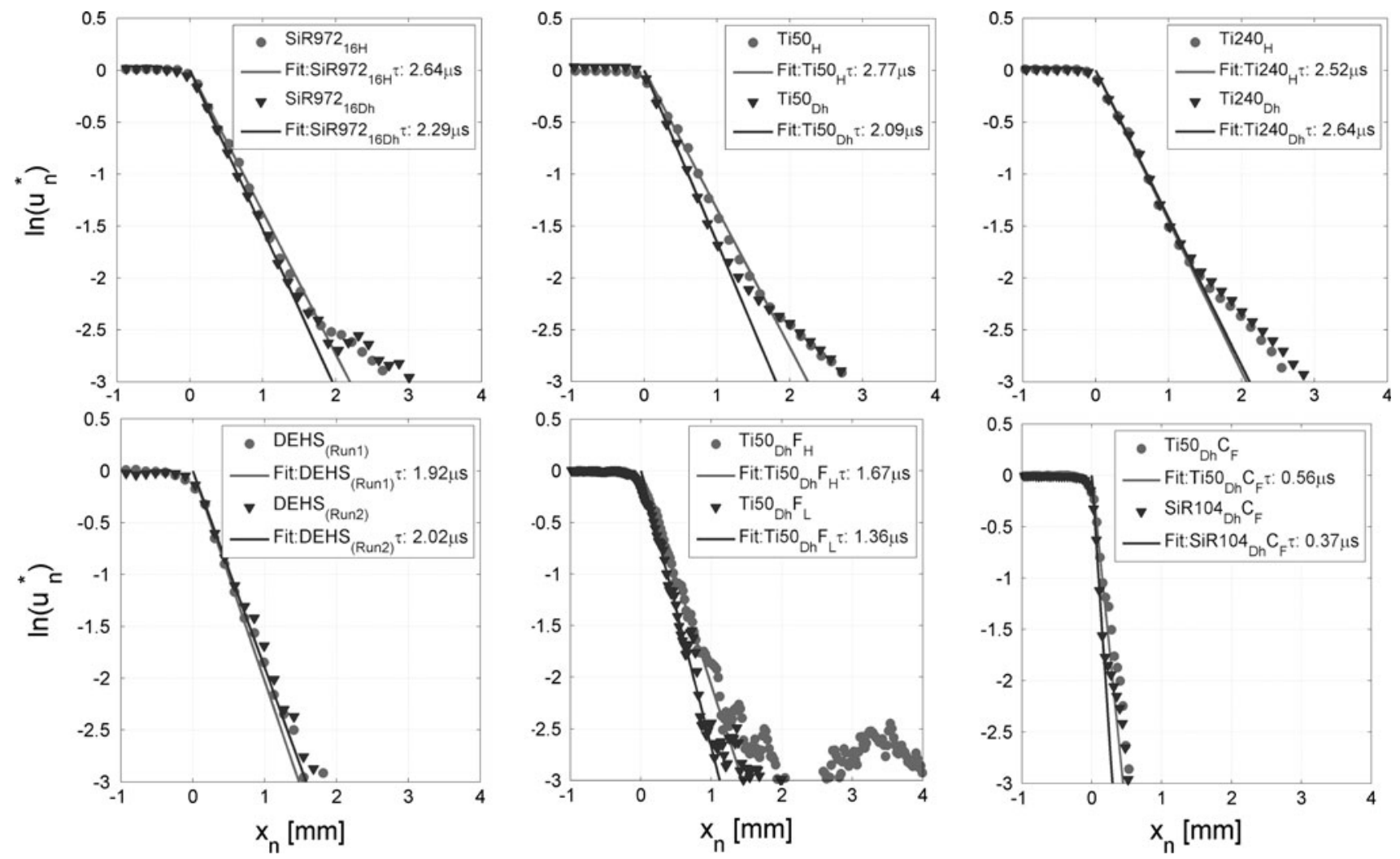

Fig. 11 Logarithmic plot of measured velocity profile across the shock for several materials with and without dehydration

Table 3 Time response of seeding materials and typical data dispersion

\begin{tabular}{|c|c|c|c|c|c|}
\hline \multirow[t]{2}{*}{ ID material } & \multirow[t]{2}{*}{ Seeder } & \multicolumn{2}{|c|}{ Mean $\tau_{p}(\mu \mathrm{s})$} & \multicolumn{2}{|l|}{$\sigma\left(\tau_{p}\right)(\mu \mathrm{s})$} \\
\hline & & Stored $\left(\tau_{H}\right)$ & De-hydrated $\left(\tau_{D h}\right)$ & Stored & De-hydrated \\
\hline DEHS & A & $1.92-2.02$ & - & $0.40-0.38$ & - \\
\hline $\mathrm{SiO}_{2} \mathrm{R} 104$ & $\mathrm{C}$ & 2.49 & 2.21 & 0.22 & 0.17 \\
\hline $\mathrm{SiO}_{2} \mathrm{R} 972$ & $\mathrm{C}$ & 2.64 & 2.29 & 0.19 & 0.28 \\
\hline $\mathrm{TiO}_{2} 14$ & $\mathrm{C}$ & 3.25 & 3.71 & 0.12 & 0.12 \\
\hline $\mathrm{TiO}_{2} 30$ & $\mathrm{C}$ & 2.56 & 2.20 & 0.11 & 0.07 \\
\hline $\mathrm{TiO}_{2} 50$ & $\mathrm{C}$ & 2.77 & 2.09 & 0.18 & 0.09 \\
\hline $\mathrm{TiO}_{2} 50$ & $\mathrm{C}_{\mathrm{F}}$ & - & 0.56 & - & 0.10 \\
\hline $\mathrm{SiO}_{2} \mathrm{R} 104$ & $\mathrm{C}_{\mathrm{F}}$ & - & 0.37 & - & 0.10 \\
\hline $\mathrm{TiO}_{2} 50$ & $\mathrm{~F}_{\mathrm{L}}$ & - & 1.36 & - & 0.05 \\
\hline $\mathrm{TiO}_{2} 50$ & $\mathrm{~F}_{\mathrm{H}}$ & - & 1.67 & - & 0.08 \\
\hline $\mathrm{TiO}_{2} 170$ & $\mathrm{C}$ & 2.50 & 3.13 & 0.13 & 0.19 \\
\hline $\mathrm{TiO}_{2} 240$ & $\mathrm{C}$ & 2.52 & 2.64 & 0.09 & 0.15 \\
\hline $\mathrm{TiO}_{2} 550$ & $\mathrm{C}$ & 2.43 & 2.78 & 0.07 & 0.22 \\
\hline
\end{tabular}

$C$ cyclone, $C_{\mathrm{F}}$ cyclone with $1 \mu \mathrm{m}$ filter, $A$ atomizer, $F$ fluidized bed dispersion, $L / H$ low/high mass flow rate that agrees with the numerical analysis presented earlier in this study. Some aero-optical effects are visible in some cases as an inflection point of the profile. Titanium and silicon dioxide particles in the crystal size range $12-50 \mathrm{~nm}$ exhibit an improved time response when dehydrated, with $\tau_{p}$ ranging from 0.4 to $2.3 \mu$ s, except for the titanium particles of the smallest crystal size. The shortest time response is obtained by using the cyclone generator with dehydrated particles combined with a $1 \mu \mathrm{m}$ particle filter. This yields a relaxation time response of $0.56 \mu$ s for the 50-nm crystal size $\mathrm{TiO}_{2}$ particles and $0.36 \mu$ s for the $\mathrm{SiO}_{2}$ with a 16-nm crystal size. The latter is a considerable improvement compared to the state-of-the-art, where typical values reported in existing literature are of 2 or $3 \mu \mathrm{s}$. 
Fig. 12 Scatter plot of individual particles velocity across the shock. Linear (left) and logarithmic (right) diagrams

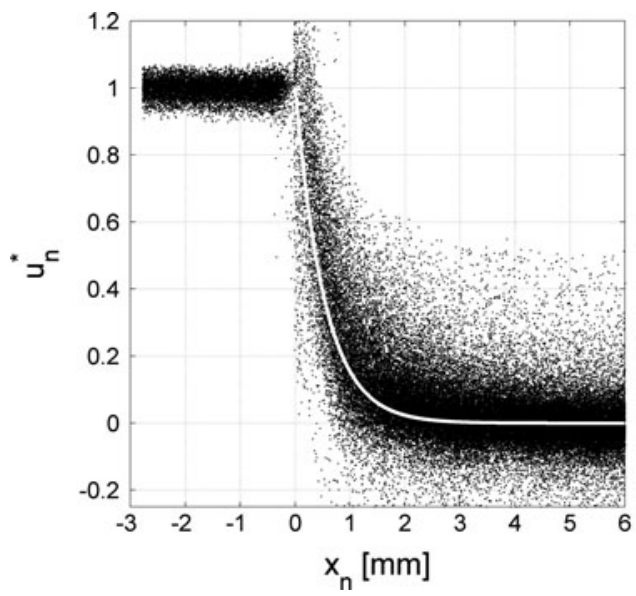

The use of a particle filter, however, strongly reduces the amount of particle tracers in the stream and the state of the filter must be constantly monitored during experiments. Unexpectedly, the performance of particles with a larger diameter appears to degrade after the drying procedure.

\subsection{PTV analysis}

In order to investigate the statistical distribution of the tracer time response, the sample of $\mathrm{TiO}_{2}$ with $50 \mathrm{~nm}$ primary crystal size after dehydration has been chosen as a reference. Particle detection in the PTV algorithm has been performed with the autocorrelation (PIV) vector field as pre-shift, restricting the search radius to 2 pixels. A total of approximately $10^{5}$ pairs occur in 200 images, in which the position of the shock has been realigned. The measured velocity is presented in Fig. 12 as a scatter plot showing that the particle velocity is dispersed around the exponential decay profile obtained from spatial auto-correlation, represented by the curve. The spreading upstream of the shock does not correspond to any physical velocity fluctuations and should therefore be interpreted as an indication of the tracking algorithm precision $(\sim 0.2$ pixels $)$. Visual inspection of the scatter plots yields a rapid increase of the (vertical) spread over the first relaxation length. The data dispersion is still rather large at $6 \mathrm{~mm}$ downstream of the shock and the upstream conditions are not attained yet. The pair's detection rate slightly decreases after the shock, partly due to the rapid deceleration causing a large velocity gradient and to a minor extent to a reduced image quality caused by aero-optical effects.

Averaging the data in the $x-u_{n}^{*}$ plane with windows of kernel $[0.2 \mathrm{~mm} \times 4 \mathrm{~m} / \mathrm{s}]$ allows computing the occurrence of particles of a given velocity at each position along the shock-normal coordinate. This is represented by contours in Fig. 13.

The results in logarithmic scale show that the particle velocity distribution is centered on the result obtained by

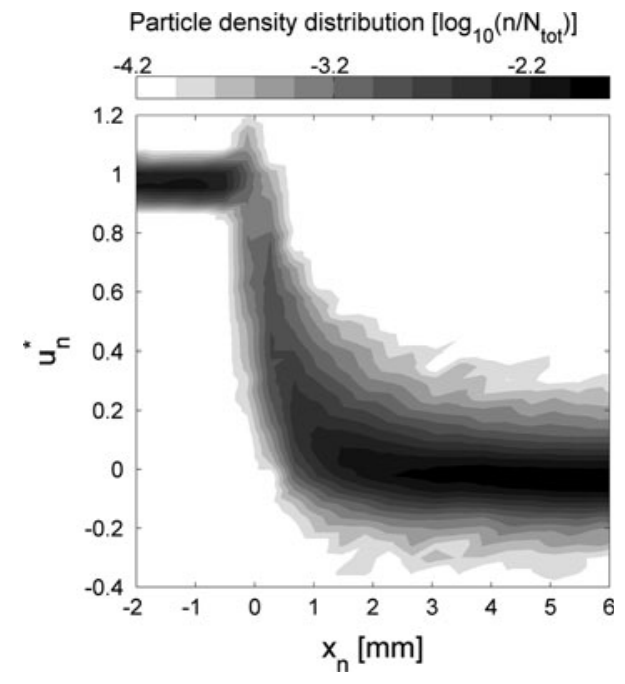

Fig. 13 Contour plot of particle occurrence in logarithmic scale

spatial auto-correlation. The availability of data in this form enables to extract profiles of the velocity distribution at different positions (Fig. 14, left). Upstream of the shock $\left(x_{n} / \xi_{\text {ref }} \leq 0\right)$, the particle velocity displays a distribution approximately Gaussian with a relatively constant standard deviation. In contrast, the particle velocity in the range $x_{n} / \xi_{\text {ref }}=[0-5]$ exhibits a wider distribution. Clearly, at $x_{n} \sim \xi_{p}$ the maximum spread is observed. Moreover, the diagram is slightly skewed with a tail towards larger values of $\tau_{p}$, corresponding to larger particles with a longer response time.

When the occurrence distribution is normalized by the maximum occurrence and rescaled with respect to a reference time response of $2 \mu$ s the diagrams obtained at different locations collapse to an acceptable extent (Fig. 14, right). Deviations are in the order of $0,1 \tau_{\text {ref }}$ (200 ns) over $5 \xi_{\text {ref. }}$. Results have been sampled in a region spanning from 0 to $5 \xi_{\text {ref }}(5 \times 0.53 \mathrm{~mm})$ downstream the shock. The peak is located around $\tau_{\text {ref }}$ confirming that the maximum occurrence corresponds to that obtained from 
Fig. 14 Left profiles of normalized occurrence at different shock-normal locations; right particles response distribution normalized to $\tau_{\text {ref }}=2 \mu \mathrm{s}$

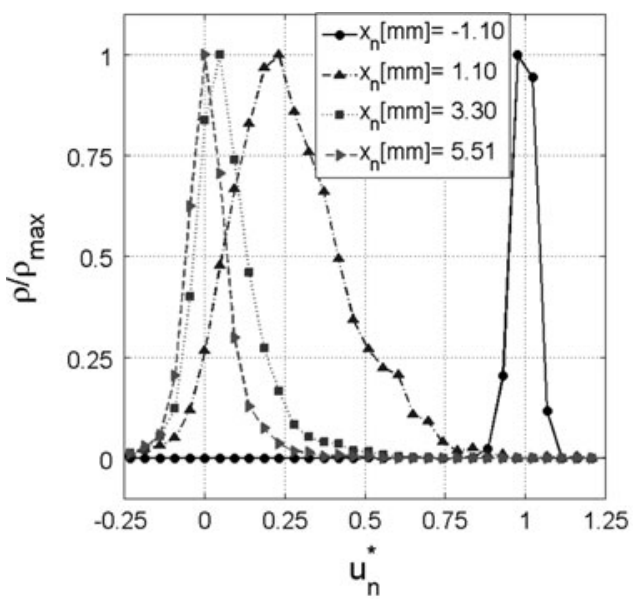

Table 4 Comparison between PTV and PIV data analyses

\begin{tabular}{llllllll}
\hline & $\mathrm{Ti} \mathrm{F}_{\mathrm{H}}$ & $\mathrm{Ti50 \textrm {C } _ { \mathrm { I } }}$ & $\mathrm{TiO}_{2} 50 \mathrm{H}$ & $\mathrm{TiO}_{2} 50 \mathrm{Dh}$ & $\mathrm{SiO}_{2} \mathrm{R}^{2} 2 \mathrm{H}$ & $\mathrm{DEHS}$ & $\mathrm{TiO}{ }_{2} 300 \mathrm{Dh}$ \\
\hline$\tau_{p} \max \pm \sigma\left(\tau_{p}\right)(\mu \mathrm{s})$ & $1.2 \pm 1.2$ & $1.0 \pm 0.8$ & $2.0 \pm 1.1$ & $1.8 \pm 1.4$ & $2.0 \pm 1.5$ & $1.8 \pm 1.4$ & $2.4 \pm 1.4$ \\
$\tau_{p} \mathrm{PIV}(\mu \mathrm{s})$ & 1.67 & 1.08 & 2.77 & 2.09 & 2.64 & 1.92 & 2.64 \\
\hline
\end{tabular}

Time response at maximum occurrence of the particles velocity distribution (upper row) and results from spatial auto-correlation (lower row)

the auto-correlation analysis. Maximum and mean values of the distributions for different seeding materials and results presented are summarized in Table 4 .

Table 4 presents the time relaxation results at maximum particle occurrence in a range spanning from 0 to $3 \mathrm{~mm}$ for a selection of the investigated seeding materials, compared with the relative auto-correlation results. The standard deviation of the distribution is calculated, and is presented as an indication, within measurement uncertainties, of the spread determined by the distribution. The values computed at the maximum $\tau_{p}$ occurrence tend to be slightly smaller than those returned by the ensemble-correlation technique. This can ascribed to the combined effect of linear combination of the ensemble-averaging and to the scattering of large particles. The spread of the distribution as well tends to reduce whenever the $\tau_{p}$ decreases for the titanium particles, suggesting that filtering and dehydration not only reduces the relaxation time but also narrows its distribution.

\section{Conclusions}

An experimental study to characterize the time response of different seeding materials has been conducted by PIV across an oblique shock wave test at Mach 2.0. An uncertainty analysis has been carried out to indicate the most important parameters governing the measurement performance. A numerical simulation of the particle motion illustrated the combined effects of finite temporal and spatial resolution as well as of the shock strength and digital resolution. It was concluded that laser pulse separation time up to $\tau_{p}$ and interrogation window up to $\xi_{p}$ are acceptable for a reliable analysis of the particle response time.

Experiments were conducted in a supersonic wind-tunnel across a planar oblique shock wave at values of TR and SR below unity by use of double exposure single frame imaging and ensemble-averaged auto-correlation analysis. The results showed that solid particles with primary crystal size below $100 \mathrm{~nm}$ yield the shortest time response when dehydrated. The $\mathrm{SiO}_{2} \mathrm{R} 104$ the $\mathrm{TiO}_{2}$ with, respectively, 16 and $50 \mathrm{~nm}$ crystal size have returned outstanding performances far below the typical value of other samples, with 0.36 and $0.56 \mu \mathrm{s}$, respectively, when dehydrated and dispersed in the TU Delft cyclone and filtered with a $3 \mathrm{M}$ 2135 P3 particle filter. The other sets of solid seeding materials yielded typical relaxation time of $2 \mu$ s and a similar behavior is followed by droplets of DEHS produced by a PIVTEC atomizer.

An attempt to characterize the statistical distribution of the particles was carried out by means of PTV analysis. Although the measurement uncertainty was higher for the PTV analysis, the results showed a physically consistent broadening of the particle tracer velocity in the relaxation region, suggesting the extent of spread in the particle tracer diameter. The statistical distribution of the relaxation time showed that the particle time response can easily vary within a factor four for a given sample material. The time response corresponding to the maximum occurrence is slightly below that measured by ensemble-averaged correlation, indicating that further improvements can be 
achieved if filters or impactors are utilized to select smaller tracers by these techniques.

Acknowledgments This work is supported by the Dutch Technology Foundation STW under the 'VIDI Innovation Impulse' programme, Grant DLR.6198. The DLR team in Cologne is acknowledged for the support in the operation of the PIVTEC fluidized bed.

Open Access This article is distributed under the terms of the Creative Commons Attribution Noncommercial License which permits any noncommercial use, distribution, and reproduction in any medium, provided the original author(s) and source are credited.

\section{References}

Cowen EA, Monismith SG (1997) A hybrid digital particle tracking velocimetry technique. Exp Fluids 22:199-211

Draper N, Smith H (1982) Applied regression analysis, 2nd edn. Darlington, $\mathrm{PB}$

Elsinga GE, van Oudheusden BW, Scarano F (2005) Evaluation of aero-optical distortion effects in PIV. Exp Fluids 39:245-256

Haertig J, Havermann M, Rey C, George A (2002) Particle image velocimetry in Mach 3.5 and 4.5 shock-tunnel flows. AIAA J 40:1056-1060

Kähler CJ, Sammler B, Kompenhans J (2002) Generation and control of tracer particles for optical flow investigation in air. Exp Fluids 33:736-742

Keane RD, Adrian RJ, Zhang Y (1995) Super-resolution particle image velocimetry. Meas Sci Technol 6:754-768

Koike S, Hidemi T, Koichi T, Mitsutomo H, Kenichi T, Goro M (2007) Correction method for particle velocimetry data based on the Stokes Drag Law. AIAA J 45:11
Lang N (2000) Investigation of the supersonic flow field around a delta wing using particle image velocimetry. In: Proceedings of the 10th international symposium on applications of laser techniques to fluid mechanics, Lisbon, PT

Mei R (1996) Velocity fidelity of flow tracer particles. Exp Fluids 22:1-13

Meinhart CD, Wereley ST, Santiago JG (2000) A PIV algorithm for estimating the time-averaged velocity fields. J Fluids Eng 122:285

Melling A (1997) Tracer particles and seeding for particle image velocimetry. Meas Sci Technol 8:1406-1416

Raffel M, Willert C, Wereley S, Kompenhans J (2007) Particle image velocimetry, a practical guide, 2nd edn. Springer, Berlin

Samimy M, Lele SK (1991) Motion of particles with inertia in a compressible free shear layer. Phys Fluids A 3:1915, 447

Scarano F, van Oudheusden BW (2003) Planar velocity measurements of a two-dimensional compressible wake. Exp Fluids 34:430-441

Schrijer FFJ, Scarano F (2007) Particle slip compensation in steady compressible flows. 7th international symposium on particle image velocimetry, Rome, Italy, September 11-14

Schrijer FFJ, Scarano F, van Oudheusden BW (2006) Application of PIV in a Mach 7 double-ramp flow. Exp Fluids 41:353-363

Schrijer FFJ, Mignoli G, Scarano F, Walpot LMGFM (2008) Experimental capsule afterbody flow investigation. In: Saccoccia $\mathrm{G}$ (ed) Proceedings of the sixth European symposium on aerothermodynamics for space vehicles

Tedeschi G, Gouin H, Elena M (1999) Motion of tracer particles in supersonic flows. Exp Fluids 26:288-296

Urban WD, Mungal MG (2001) Planar velocity measurements in compressible mixing layers. J Fluid Mech 431:189-222

Wereley S, Meinhart C (2001) Second-order accurate particle image velocimetry. Exp Fluids 31:258-268

Westerweel J (1997) Fundamentals of digital particle image velocimetry. Meas Sci Technol 8:1379-1392 